\title{
Improved biocompatibility but limited graft survival after purification of alginate for microencapsulation of pancreatic islets
}

\author{
P. De Vos ${ }^{1}$, B .J . De H aan ${ }^{1}$, G.H.J. Wolters ${ }^{1}$, J.H . Strubbe ${ }^{2}$, R. Van Schilfgaarde ${ }^{1}$ \\ ${ }^{1}$ Surgical Research Laboratory, Department of Surgery, University of Groningen, Groningen, The Netherlands \\ ${ }^{2}$ Department of Animal Physiology, University of Groningen, Haren, The Netherlands
}

Summary Graft failure of alginate-polylysine microencapsulated islets is often interpreted as the consequence of a non-specific foreign body reaction against the microcapsules, initiated by impurities present in crude alginate. The aim of the present study was to investigate if purification of the alginate improves the biocompatibility of alginate-polylysine microcapsules. Alginate was purified by filtration, extraction and precipitation. Microcapsules prepared from crude or purified alginate were implanted in the peritoneal cavity of normoglycaemic AO-rats and retrieved at 1, 2, 3, 6, 9, and 12 months after implantation. With crude alginate, all capsules were overgrown within 1 month after implantation. With purified alginate, however, the portion of capsules overgrown was usually less than $10 \%$, even at 12 months after implantation. All recipients of islet allografts in capsules prepared of purified alginate became normoglycaemic within 5 days after implantation, but hyperglycaemia reoccurred after 6 to
20 weeks. With intravenous and oral glucose tolerance test, all recipients had impaired glucose tolerance and insulin responses were virtually absent. After graft failure, capsules were retrieved (80-100\%) by peritoneal lavage. Histologically, the percentage of overgrown capsules was usually less than $10 \%$ and maximally $31 \%$. This small portion cannot explain the occurrence of graft failure. The immunoprotective properties of the capsules were confirmed by similar if not identical survival times of encapsulated islet allo- and isografts. Our results show that purification of the alginate improves the biocompatibility of alginate-polylysine microcapsules. Nevertheless, graft survival was still limited, most probably as a consequence of a lack of blood supply to the encapsulated islets. [Diabetologia (1997) 40: 262-270]

Keywords Purification, alginate, graft function, glucose tolerance, microencapsulation.
Alginate-polylysine microencapsulation allows for successful transplantation of allo- and xenogenic islets but graft function is still limited [1-6]. Usually, graft failure is interpreted as the consequence of a

Received: 20 August 1996 and in revised form: 25 November 1996

Corresponding author: Dr. P. De Vos, Surgical Research Laboratory, Department of Surgery, University of Groningen, Bloemsingel 1, NL-9713 BZ Groningen, The Netherlands A bbreviations: KRH, Krebs-Ringer-Hepes; BSA, bovine serum albumin; $\mathrm{Ca}^{2+}$-free $\mathrm{KRH}, \mathrm{Ca}^{2+}$-free Krebs-Ringer-Hepes buffer; PLL, poly-l-lysine; IVGTT, intravenous glucose tolerance test. non-specific foreign body reaction against the microcapsules resulting in progressive fibrotic overgrowth of the capsules [5-11] and necrosis of the islets.

Alginate is a polysaccharide composed of mannuronic and guluronic acids. Mannuronic acid has been identified as the major initiator of the foreign body reaction by some [7], while others found guluronic acid to be associated with more severe fibrotic overgrowth [8]. Coating of the capsules with additional layers of alginate has been found to be associated with less severe fibrotic overgrowth [11] which suggests a role for polylysine in the initiation of the foreign body reaction. All these studies, however, were performed with alginates which had not been purified. 
Alginate is a crude product extracted from alga and is contaminated with different inflammation provoking components [12] of which only polyphenols and endotoxins have been identified. Varying degrees of purity may explain the controversies in studies addressing the cause of the bioincompatibility of alginate-poly-L-lysine (PLL) capsules.

The aim of the present study in rats was to investigate the effect of purification of alginates on the biocompatibility of alginate-PLL microcapsules. Therefore, a purification procedure for alginate was developed. Empty capsules prepared of either crude or purified alginate were implanted in the peritoneal cavity and retrieved after varying time intervals for histological evaluation of the degree of the fibrotic response against the capsule grafts. Furthermore, we investigated the function of microencapsulated islet allografts and the capsular overgrowth of encapsulated iso- and allografts when purified alginate was applied.

\section{Materials and methods}

D esign of the study. Batches of empty microcapsules were divided into six portions of approximately $2 \mathrm{ml}$ and subsequently implanted into the peritoneal cavity of six normoglycaemic AO rats. Of these six rats, one was subjected to peritoneal lavage at 1,2, 3, 6, 9 or 12 months after implantation. Similar volumes of capsules were implanted in Biobreeding (BB)-rats in order to exclude rat strain dependency of the reaction against the capsules. From these rats the capsules were retrieved by peritoneal lavage at 1 month postimplant only.

The total volume of the capsules recovered by peritoneal lavage was related to the initial volume of the implanted graft. The number of recovered capsules with fibrotic overgrowth was assessed by microscopy of histological sections of the capsules. This fraction of overgrown capsules was expressed as the percentage of the total number of recovered capsules.

Diabetic AO-rats served as recipients of capsules prepared from purified alginate containing either AO (isografts) or Lewis-islets (allografts). Islets were cultured before encapsulation in order to reduce contamination of the grafts with exocrine tissue, ducts or damaged cells. Graft endocrine volume was at least $10 \mu \mathrm{l}$, which is approximately equal to the endocrine volume of the normal rat pancreas [13]. Blood glucose was determined at regular time intervals. Recipients with blood glucose levels lower than $8.4 \mathrm{mmol} / \mathrm{l}$ were considered normoglycaemic. One or two weeks after the reoccurrence of hyperglycaemia, microcapsules were retrieved from the diabetic recipients by peritoneal lavage and processed for histological examination. At 4 to 6 weeks after implantation of successful encapsulated islet allografts the rats were provided with two cardiac catheters implanted via the right and left jugular vein for blood sampling and infusion of glucose, respectively, in unanaesthetized and freely moving rats [14, 15]. Subsequently, the function of encapsulated islet allografts was tested by an intravenous glucose tolerance test (IVGTT) and by a meal test $[6,13]$. Normal rats served as controls.

A nimals and induction of diabetes. Male inbred Albino Oxford $(\mathrm{AO} / \mathrm{G})$ rats were obtained from the Central Animal Laboratory of Groningen, inbred Lewis rats were obtained from
Harlan CPB, Zeist, The Netherlands, and BB/Org-rats were obtained from the Central Animal Facility of Erasmus University, Rotterdam, The Netherlands.

$\mathrm{AO} / \mathrm{G}-$ rats and Lewis-rats weighing 300-350 g served as donors. AO/G-rats weighing 290-320 g were used as recipients of encapsulated islet grafts. Diabetes was induced in these rats by injection of $75-90 \mathrm{mg} / \mathrm{kg}$ of streptozotocin (Zanosar, Upjohn Co., Kalamazoo, Mich., USA) via the tail vein. A second injection of $70-90 \mathrm{mg} / \mathrm{kg}$ of streptozotocin was administered when at 10-14 days after the first injection the blood glucose level was less than $20 \mathrm{mmol} / 1$. Only animals with severe weight loss, polyuria, polydipsia and fasting blood glucose levels exceeding $20 \mathrm{mmol} / \mathrm{l}$ over a period of at least 4 weeks were used as recipients. At the time of peritoneal lavage a biopsy was taken from the tail of the pancreas. If not mentioned otherwise the rats had less than $5 \%$ of the normal number of beta cells present in their pancreas. As shown in one of our previous studies [16], the circulating insulin levels in these diabetic rats never exceeded $24 \mathrm{pmol} / \mathrm{l}$.

I slet isolation. Islets were isolated as previously described [17]. Briefly, under ether anaesthesia, the abdomen was opened and the common bile duct was cannulated under non-sterile conditions. The donor pancreas was distended with $10 \mathrm{ml}$ sterile Krebs-Ringer-Hepes supplemented with $25 \mathrm{mmol} / \mathrm{l}$ Hepes buffer (KRH) and containing $10 \%$ bovine serum albumin (BSA). Subsequently the pancreas was excised, and brought into a laminar flow cabinet. All further procedures were performed under sterile conditions.

The pancreas was chopped, digested using a two-stage incubation of $20 \mathrm{~min}$ at $37^{\circ} \mathrm{C}$ with successively 1.0 and $0.7 \mathrm{mg} / \mathrm{ml}$ collagenase (Sigma type XI; Sigma, St. Louis, Mo., USA). Islets were separated from exocrine tissue by centrifugation over a discontinuous dextran gradient [18] and further purified by handpicking.

The total islet volume obtained by the isolation procedure was determined by measuring the diameters [13] of islets in a $4 \%$ aliquot of the islet suspension. Subsequently, the total volume was calculated, assuming the islets to be perfect spheres. In each experiment we used four to five donors from which we isolated a total of 3500-4200 islets which corresponds to $10-15 \mu l$ of islet tissue.

The islets were cultured in non-treated petri-dishes (Greiner, Alphen a/d Rijn, The Netherlands) in portions of 100 islets per $25 \mathrm{~cm}^{2}$ for 19 to $44 \mathrm{~h}$ in CMRL1066 (containing $10 \%$ fetal calf serum (FCS, Gibco, Breda, The Netherlands), $8.3 \mathrm{mmol} / \mathrm{l}$ glucose and $10 \mathrm{mmol} / 1 \mathrm{Hepes}$ and $1 \%$ penicillium/ streptomycin) at $37^{\circ} \mathrm{C}$ in humidified air containing $5 \% \mathrm{CO}_{2}$. After this culture period the total islet volume was assessed again.

The diameter of the islets was measured with a dissection microscope with a fluorescent illuminator (Bausch and Lomb BVB-125, and 31-33-66, Rochester, NY, USA) equipped with an ocular micrometer with an accuracy of $25 \mu \mathrm{m}$.

Purification of alginate. Crude sodium alginate (Keltone LV; Kelco International, London, UK) was dissolved at $4{ }^{\circ} \mathrm{C}$ in a $1 \mathrm{mmol} / \mathrm{l}$ sodium EGTA solution to a $1 \%$ solution under constant stirring, and filtered over, successively, 5.0, 1.2, 0.8, and $0.45 \mu \mathrm{m}$ filters (Schleicher \& Schüll, 's-Hertogenbosch, The Netherlands). During this filtration step, all visible aggregates were removed, and the solution lost its original yellow colour and became translucent.

Next, the $\mathrm{pH}$ of the solution was lowered to 3.5 under constant monitoring by addition of $2 \mathrm{~N} \mathrm{HCl}$ plus $20 \mathrm{mmol} / \mathrm{l} \mathrm{NaCl}$. The solution was kept on ice to prevent hydrolysis of alginate. The further slow lowering from 3.5 to 1.5 was associated with 
gradual precipitation of alginate as alginic acid (precipitation curves not shown) [19]. Routinely, the solution was brought at a $\mathrm{pH}$ of 2.0 and subsequently filtered over a Buchner funnel (pore size $1.5 \mathrm{~mm}$ ) to wash out non-precipitated contaminants. To extend the wash-out of non-precipitated contaminants, the precipitate was brought in $0.01 \mathrm{~N} \mathrm{HCL}$ plus $20 \mathrm{mmol} / \mathrm{l} \mathrm{NaCl}$, vigorously shaken, and filtered again over the Buchner funnel. This washing procedure was performed three times.

Then, proteins were removed by extraction with chloroform/butanol [20]. The alginic acid was suspended in $100 \mathrm{ml}$ of $0.01 \mathrm{~N} \mathrm{HCl}$ plus $20 \mathrm{mmol} / 1 \mathrm{NaCl}$ and supplemented with $20 \mathrm{ml}$ chloroform and $5 \mathrm{ml} \mathrm{1-butanol}$. The mixture was vigorously shaken for $30 \mathrm{~min}$ and filtered over the Buchner funnel. This chloroform/butanol extraction was performed three times. Next, the alginic acid was brought in water and slowly dissolved by gradually raising the $\mathrm{pH}$ to 7.0 by the slow addition of $0.5 \mathrm{~N} \mathrm{NaOH}$ plus $20 \mathrm{mmol} / \mathrm{l} \mathrm{NaCl}$ over a period of at least 1 hour. The alginate solution obtained was subjected to a chloroform/butanol extraction to remove those proteins which can only be dissolved in chloroform/butanol at neutral $\mathrm{pH}$ [20]. The solution was vigorously shaken in a mixture of chloroform (20 ml at each $100 \mathrm{ml}$ alginate solution) and 1-butanol (5 ml at each $100 \mathrm{ml}$ alginate solution) for $30 \mathrm{~min}$. The mixture was centrifuged for 3-5 min at $3000 \mathrm{rev} / \mathrm{min}$, which induced the formation of a separate chloroform/butanol phase which was removed by aspiration. The extraction was repeated once.

The final step was precipitation of the alginate with ethanol $[21,22]$. To each $100 \mathrm{ml}$ of alginate solution we added $200 \mathrm{ml}$ absolute ethanol (Ethylalcohol, absolute; NedAlco, Bergen op Zoom, The Netherlands). After an incubation period of 10 min all alginate had precipitated. It was filtered over the Buchner funnel and washed two times with absolute ethanol. Finally, the alginate was washed three times with ethylether and freeze-dried overnight.

Usually, the purification run was started with $10 \mathrm{~g}$ of alginate of which 6-7.5 g remained at the end of the procedure. The transplant studies were performed with three different Keltone LV lot numbers. This purification procedure did not influence the characteristics of the alginate, since analysis [23, $24]$ of the composition of the alginate before and after purification showed no changes in its guluronic acid and mannuronic acid content. It was always composed of $40 \%$ guluronic acid and $60 \%$ mannuronic acid.

Formation of islet-containing and empty capsules. After culture, islets were washed three to five times with RPMI containing $10 \%$ FCS and subsequently suspended in sterile filtered $(0.2 \mu \mathrm{m}$ filtration) $3 \%$ purified alginate solution. The alginate solution was converted into droplets using an air-driven droplet generator as previously described [25]. Polylysine-alginate encapsulation was performed as described elsewhere [26]. Briefly, the alginate droplets were transformed to alginate beads by gelling in a $100 \mathrm{mmol} / 1 \mathrm{CaCl}_{2}(10 \mathrm{mmol} / \mathrm{l} \mathrm{HEPES}$, $2 \mathrm{mmol} / \mathrm{l} \mathrm{KCl}$ ) solution for at least $5 \mathrm{~min}$. After gelation the beads had a diameter of $450-550 \mu \mathrm{m}$. Subsequently, the Ca-alginate beads were suspended for $1 \mathrm{~min}$ in KRH buffer containing $2.5 \mathrm{mmol} / 1 \mathrm{CaCl}_{2}$. A PLL membrane was formed by suspending the alginate beads in $0.1 \%$ PLL solution for $10 \mathrm{~min}$ (poly-L-lysine-HCl, $\mathrm{M}_{\mathrm{r}}$ : 22000, Sigma). Non-bound PLL was removed by three successive washings during $3 \mathrm{~min}$ with $\mathrm{Ca}^{2+}$-free KRH containing $135 \mathrm{mmol} / \mathrm{l} \mathrm{NaCl}$. The outer alginate-layer was subsequently applied by 5 min incubation in 10 times diluted alginate solution. In order to liquify their inner cores, the microcapsules were suspended in $1 \mathrm{mmol} / \mathrm{l}$ EGTA in $\mathrm{Ca}^{2+}$-free $\mathrm{KRH}$ for $10 \mathrm{~min}$, and washed three times with $\mathrm{KRH}$ containing $2.5 \mathrm{mmol} / \mathrm{l} \mathrm{CaCl}_{2}$. Finally, the capsules had a diameter of $600-700 \mu \mathrm{m}$ and were impermeable for substances larger than $100 \mathrm{kDa}$, as measured by diffusion into the capsules of lectins with varying molecular weights [27].

Viability of the islet tissue before transplantation was confirmed in the majority of the experiments by applying the acridine orange (Sigma)/propidium iodide (Sigma) viability assay [28] on portions of 20-100 islet-containing capsules. We always found more than $85 \%$ of the islet tissue to be vital.

Implantation and explantation of islet containing and empty capsules. Under ether anaesthesia, the microencapsulated islets or empty capsules were injected into the peritoneal cavity with a 16 gauge cannula via a small incision $(3 \mathrm{~mm})$ in the linea alba. The abdomen was closed with a two-layer suture. The implanted volumes varied between 1.5 and $2.5 \mathrm{ml}$.

Peritoneal lavage was performed by infusing $20 \mathrm{ml} \mathrm{KRH}$ through a $3-\mathrm{cm}$ midline incision into the peritoneal cavity and subsequent flushing of the abdomen above a $50 \mathrm{ml}$ centrifuge tube. The abdominal organs were flushed two to three times with KRH.

G lucose tolerance tests. IVGTTs were performed by infusion of $200 \mathrm{mg}$ glucose at a rate of $10 \mathrm{mg} / \mathrm{min}$. The meal tests were carried out by offering the animal $2 \mathrm{~g}$ rat chow (containing $53 \%$ carbohydrate, $20 \%$ protein, $5 \%$ fat and $22 \%$ other constituents [minerals, cellulose, water]), mixed with $2 \mathrm{ml}$ water. The animals had been habituated to consume the meal within 5 min.

In both tests, blood samples were taken at $10 \mathrm{~min}$ and immediately before the start of the tests, and at 1, 3, 5, 7, 10, $15,20,25,30,45,60,75,90,105$, and $120 \mathrm{~min}$ after the start of the tests. Blood lost during sampling was replaced by transfusing blood from normal donor rats after every blood sample.

In some recipients, glucose tolerance could not or not completely be tested as a consequence of technical complications such as death related to cannulation surgery or occlusion of the catheters, or refusal to eat more than $70 \%$ of the test meal.

Chemical determinations. Glucose concentrations during the glucose tolerance testing were determined in whole blood by a ferricyanide method with a Technicon autoanalyser. The glucose concentration in blood obtained at weekly intervals was determined with glucose test tapes (Reflolux; Boeringer Mannheim, Mannheim, FRG). Plasma insulin was measured by a radioimmunoassay (Linco, Rat Insulin Ria Kit, St. Charles, MO, USA). Glycosylated hemoglobulin was measured by applying an affinity based chromatography assay (Glyc-Affin GHB, ISOLABinc., Ohio, USA).

$\mathrm{H}$ istology and immunohistochemistry of recovered material. Half of the volume of recovered capsules and biopsies of capsules stuck to abdominal organs were fixed in pre-cooled $2 \%$ paraformaldehyde, buffered with $0.05 \mathrm{~mol} / \mathrm{l}$ phosphate, and processed for glycol methacrylate embedding [29]. Sections were prepared at $2 \mu \mathrm{m}$, stained with Romanovsky-Giemsa stain, and applied to assess the degree of capsular overgrowth, which was quantified by expressing the number of recovered capsules with overgrowth as the percentage of the total number of recovered capsules.

The other half of recovered microcapsules and the pancreas biopsies were fixed in Bouin's solution for paraffin processing, sectioned at $5 \mu \mathrm{m}$, and stained with aldehyde fuchsin. In these sections, the presence or absence of viable beta cells was determined. 


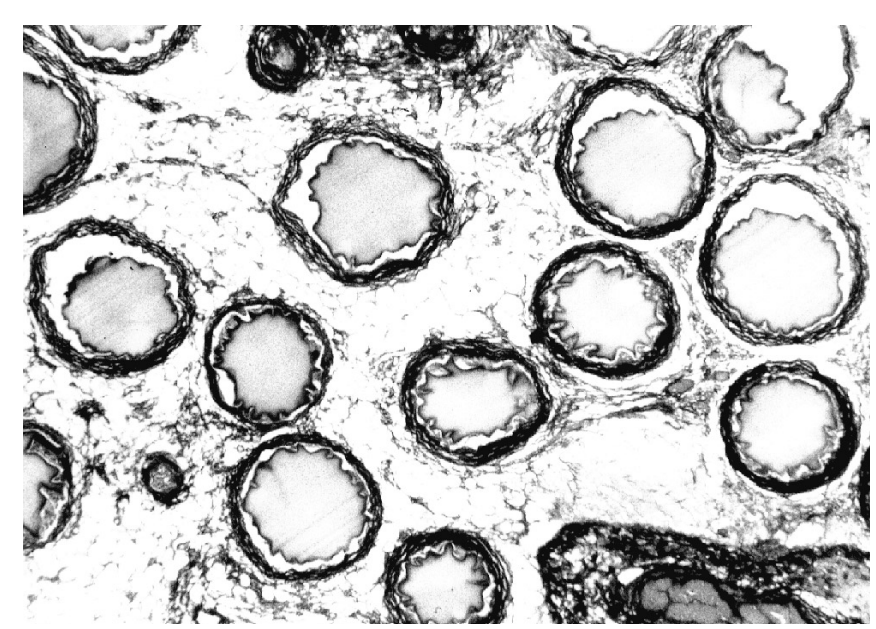

Fig. 1. Alginate-polylysine capsules prepared of crude alginate, 1 month after implantation. The capsules are adherent to the omentum. (GMA-embedded histological section, Syrius-Red stain, original magnification $\times 50$ )

Statistical analysis. Statistical evaluation was performed using the Mann Whitney U test. Values are expressed as mean \pm SEM. Only $p$ values less than 0.05 were considered to indicate a statistically significant difference.

\section{Results}

E mpty capsules prepared from crude and purified alginate. Within 1 month post-implant, all empty capsules prepared of crude alginates were overgrown by fibrotic tissue and surrounded by collagen deposits (Fig.1). The capsules were found to be adherent to the omentum and the liver capsule. Up to 18 months after implantation, we still found capsules at these sites, and found no indications of biodegradability of the capsules.

Occasionally, we found a portion of the empty capsules prepared from purified alginate to be adherent to the omentum and the liver capsule. The majority of capsules, however, was freely floating in the abdominal cavity without adhesion to the abdominal organs. The free capsules could readily be flushed out of the peritoneal cavity, resulting in an explantation of $80-100 \%$ up to 1 year post-implant (Fig. 2). Of these recovered capsules, the mean percentage of capsules with overgrowth remained usually well below $10 \%$ with monthly assessments during the year. There was one exception at 3 months post-implant $(12.6 \pm 9 \%)$ as the consequence of one single recipient with $30 \%$ overgrowth.

In histological sections of explants we recurrently observed that some capsules contained defects which were surrounded by immune cell elements with the morphological appearance of macrophages (Fig. 3). This cellular infiltration of defect capsules, however, was a temporary phenomenon since after 2 months

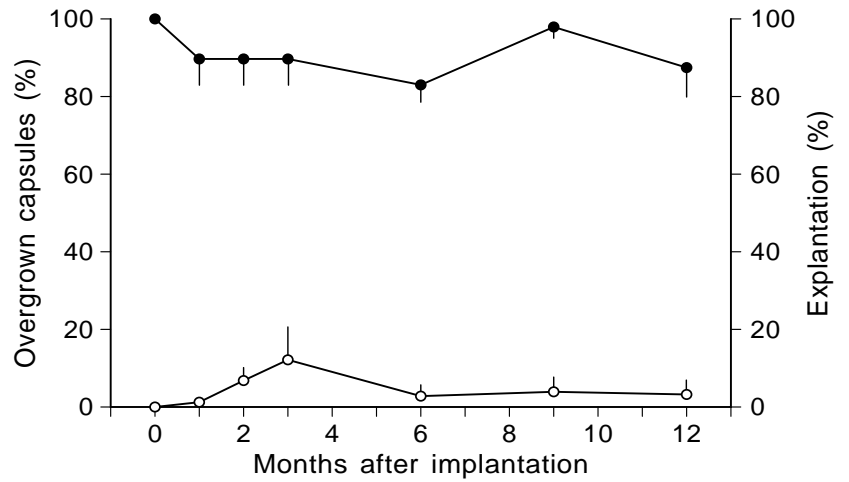

Fig. 2. Percentage capsules explanted (O) and overgrown by fibrotic tissue $(\bigcirc)$. Capsules were prepared of purified alginates and implanted in the peritoneal cavity of normoglycaemic AO-rats. Every time point represents the average of the results obtained in four animals

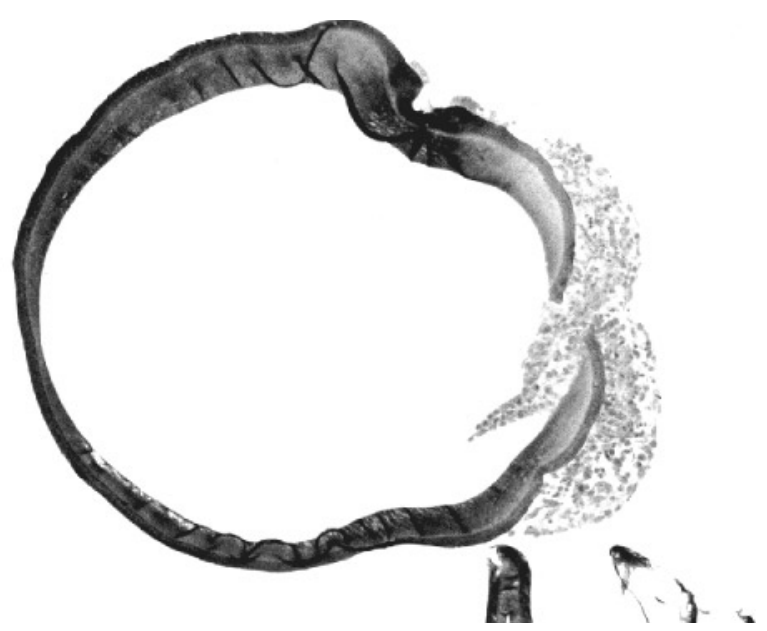

Fig. 3. Empty alginate-polylysine capsule prepared from purified alginate, 1 month after implantation. The capsule contains a defect and is overgrown by macrophages. (GMA-embedded histological section, Romanowsky-Giemsa stain, original magnification $\times 150$ )

only overgrowth composed of several layers of fibroblasts surrounding the capsule was observed.

One series of 6 animals was excluded from the study since a severe inflammatory reaction had occurred resulting in sepsis and death of two recipients. This inflammatory reaction was induced by bacterial contamination of the purified alginate.

In order to exclude that the mild reaction towards the capsules prepared from purified alginate is specific for the AO-rat strain we also implanted empty capsules in the peritoneal cavity of four prediabetic BB-rats which reportedly are high responders toward alginate-PLL capsules [4, 8]. Also in the BB-rats, 1 month after implantation, we observed the majority of capsules to be freely floating in the peritoneal cavity. By peritoneal lavage $79 \pm 9 \%$ of the capsules could be retrieved of which a portion of $9.3 \pm 0.9 \%$ was overgrown by fibrotic tissue. 


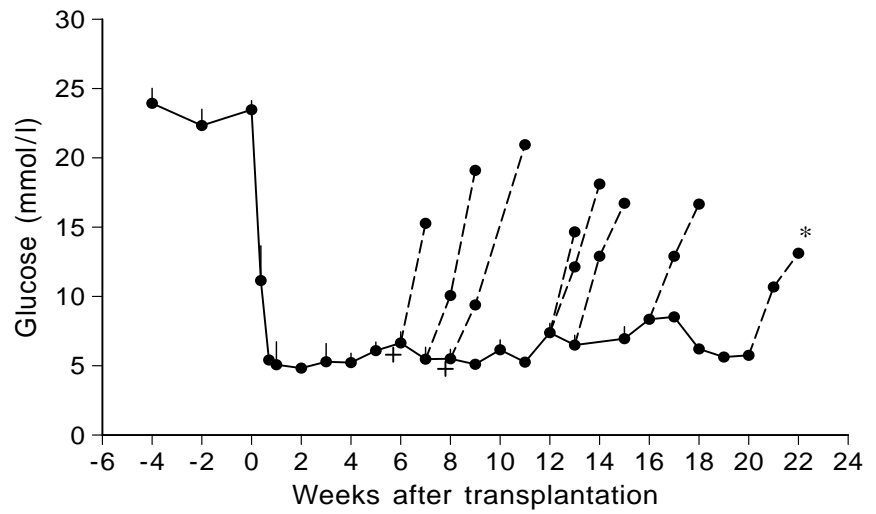

Fig. 4. Non-fasting blood glucose concentrations in streptozotocin diabetic AO-rats $(n=10)$ after implantation of islet allografts microencapsulated in capsules prepared of purified alginate. (-) animals returning to hyperglycaemia, + , animals which died of a technical complication, * animals with 5-10\% vital beta cells in their native pancreas. Values represent mean \pm SEM

Islet allografts encapsulated with purified alginate. Transplantation of encapsulated islets was restricted to experiments with purified alginate since in a previous study [27] we found that fibrotic responses similar to that observed against the crude alginate were always associated with early graft failure.

Fig. 5 A , B. Blood glucose and plasma insulin levels after intravenous glucose infusion $(10 \mathrm{mg} / \mathrm{min})(\mathrm{n}=4)(\mathbf{A})$ and after spontaneous ingestion of a meal $(n=5)(B)$ in AO- recipients of microencapsulated islet allografts $(\mathcal{)})$ and in normal control rats $(\bigcirc)$
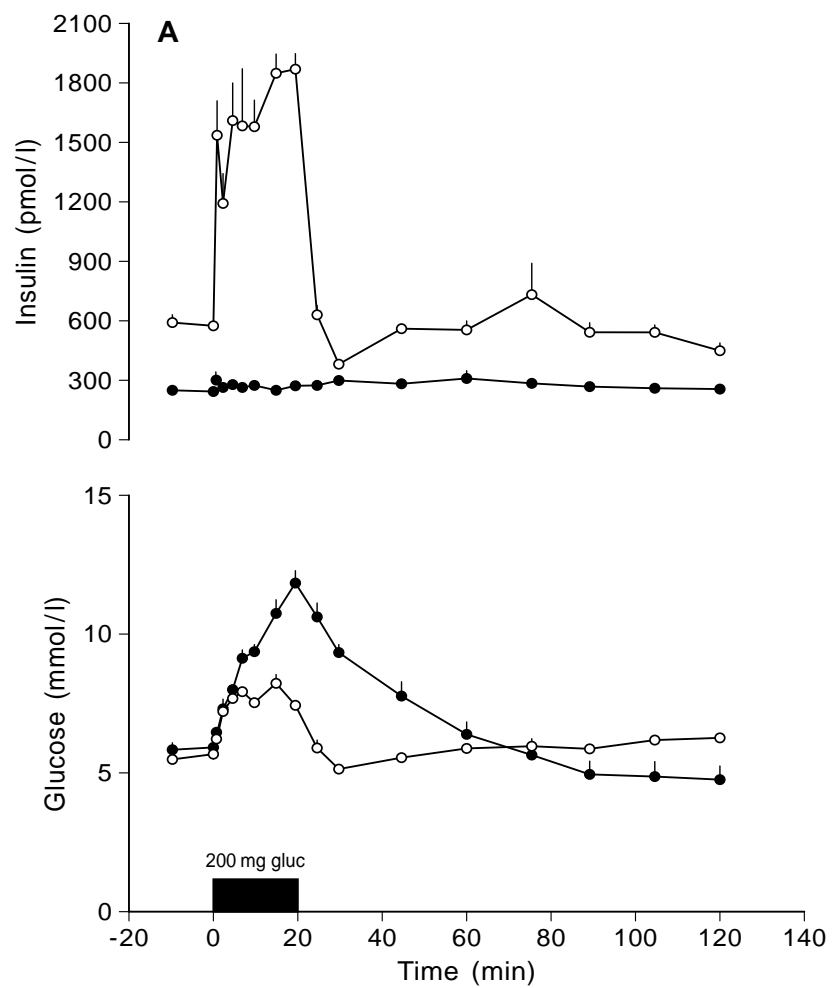

Streptozotocin diabetic AO recipients of allogenic islets encapsulated in purified alginate became normoglycaemic in 10 out of 13 cases. The 10 successfully transplanted rats became normoglycaemic within 5 days after implantation and remained normoglycaemic for 6 to 20 weeks (Fig. 4). As shown in our previous study, AO recipients never became normoglycaemic after transplantation of $10 \mu l$ of non-encapsulated Lewis islet tissue [6].

Prior to glucose tolerance tests, basal insulin levels in recipients of microencapsulated islet allografts were much lower than in normal control rats. These low insulin levels did not correspond with the concomitant basal glucose levels which were found to be similar in recipients and control rats (Fig. 5). The glucose challenge did not initiate an elevation of plasma insulin in rats with microencapsulated islets, while normal control rats had a clear insulin response during both the IVGTT and the meal test. The concomitant glucose levels in recipients of encapsulated islet allografts reached higher levels and returned more slowly to basal levels than in normal control rats.

The results of the meal tests in two recipients were excluded from the calculations of the mean since these two recipients, unlike the other recipients, showed disparate results with intravenous and oral glucose challenge. Their response to IVGTT was similar to that of other recipients. But with meal tests 1 week later, we observed much higher peak glucose levels and no re-establishment of basal glucose levels during the test, in spite of normoglycaemic basal glucose levels and regular insulin levels prior to the test.
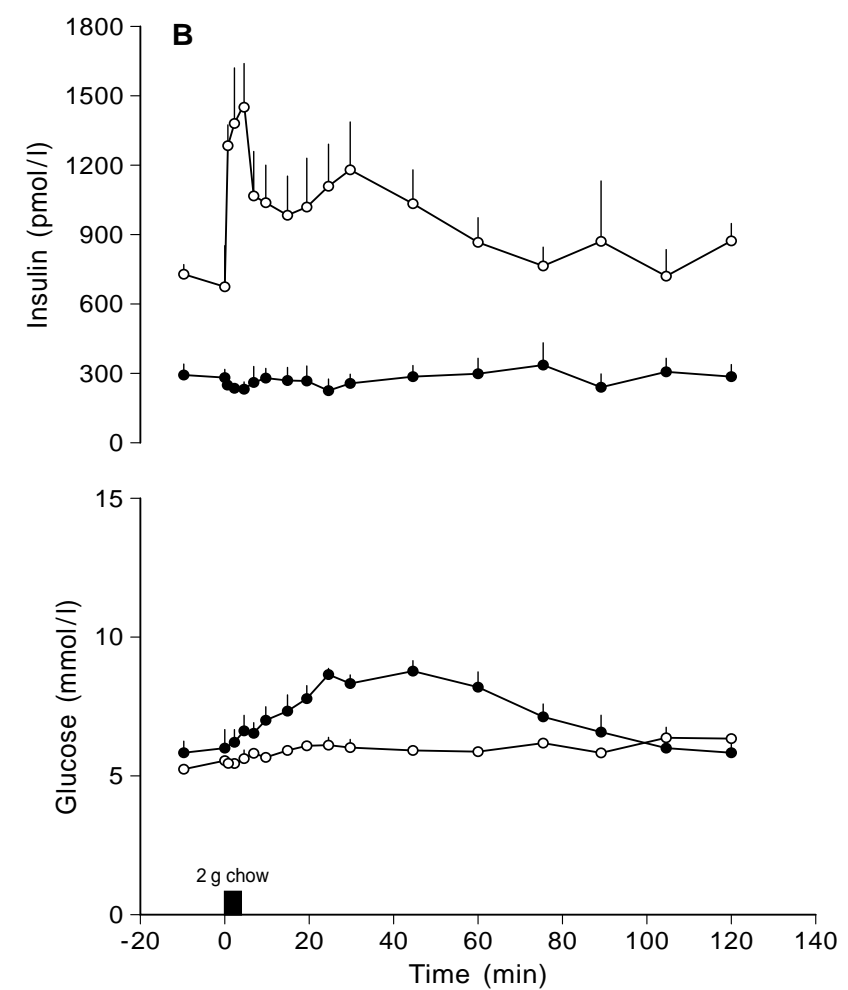


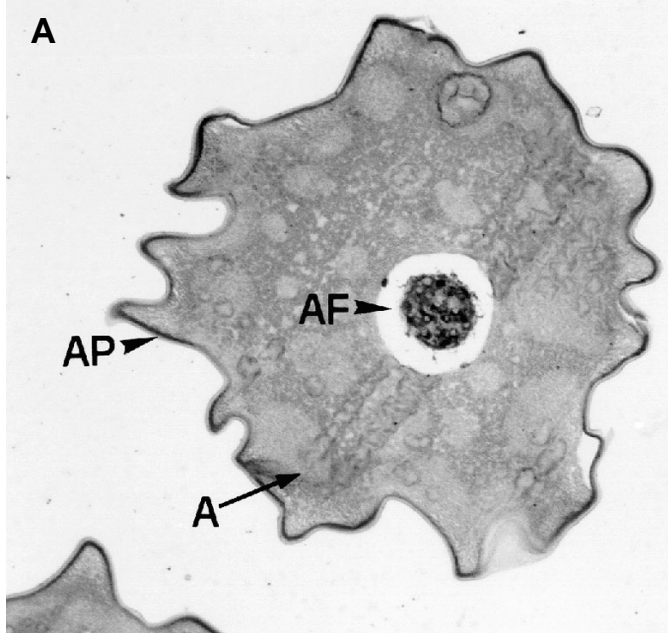

Fig. 6 A , B. Alginate-polylysine encapsulated islet, after allotransplantation. (A) An example of an islet surrounded by an alginate free ring, 4 weeks after implantation (paraffin-embedded histological section, Aldehyde-fuchsin stain, original magnification $\times 100)$. AP, alginate polylysine; $A F$, alginate free zone; A, alginate. (B) An islet positively stained for insulin, 14 weeks after transplantation (GMA-embedded histological section, original magnification $\times 200)$. Arrow indicates necrotic zone

After another week, both animals had completely returned to hyperglycaemia with elevated basal glucose levels.

In spite of disturbed glucose tolerance during the glucose challenge, we found in all successfully transplanted recipients, the percentage glycosylated haemoglobulin to be reduced from a pretransplant level of $10.4 \pm 1.2 \%$ to $4.6 \pm 0.4 \%$ within 3 weeks after transplantation. This post-transplant glycosylated haemoglobulin percentage was not significantly different from the percentage glycosylated haemoglobulin in control rats, which was $4.3 \pm 0.1 \%$.

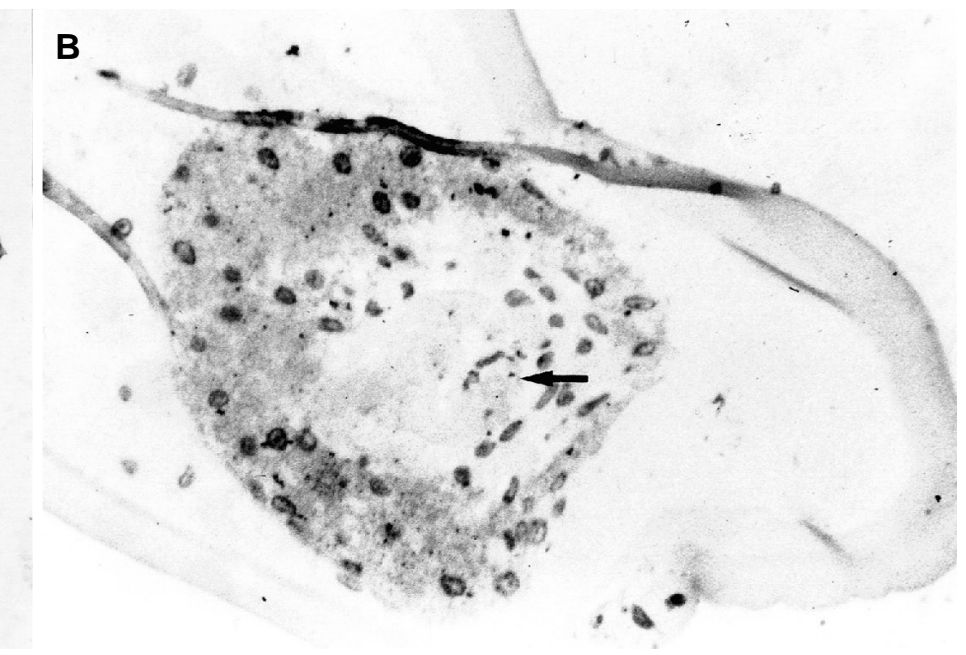

$\mathrm{H}$ istology of islet allografts encapsulated with purified al ginate. Two recipients were normoglycaemic when they died 6 and 8 weeks after transplantation (Fig.4). From these two, we could retrieve 60 and $100 \%$ of the capsules, 2 and $31 \%$ of which were overgrown by fibrous tissue, respectively. The microcapsules were found to contain many viable islets staining positive for insulin. The islets, however, had smaller diameters than before transplantation as indicated by the presence of an alginate free ring around the encapsulated islets (Fig. 6.A).

The other eight recipients were killed approximately 1 or 2 weeks after the reoccurrence of hyperglycemia. In these animals, the majority of capsules was found to be freely floating in the peritoneal cavity without any cellular adhesion. The portion of overgrown capsules in these eight recipients was always similar or even smaller than the portion of overgrown capsules found in the other two (Table 1). There was no relation between the duration of graft function and the number of capsules overgrown by fibrotic tissue. After graft failure we found nonovergrown capsules with non-viable islet remnants.

Table 1. Recovery rates and percentage of capsules with overgrowth at varying time intervals after implantation of microencapsulated rat allografts in streptozotocin diabetic AO-rats

\begin{tabular}{|c|c|c|c|}
\hline $\begin{array}{l}\text { Time (weeks) between implantation } \\
\text { and peritoneal lavage }\end{array}$ & $\begin{array}{l}\text { State of glycaemia immediately } \\
\text { preceding peritoneal lavage }^{\mathrm{a}}\end{array}$ & Percentage of retrieval & $\begin{array}{l}\text { Percentage of capsules } \\
\text { with overgrowth }\end{array}$ \\
\hline $6^{\mathrm{b}}$ & normo & 60 & 2 \\
\hline $8^{b}$ & normo & 100 & 31 \\
\hline 7 & hyper & 75 & 14 \\
\hline 8 & hyper & 100 & 6 \\
\hline 12 & hyper & 90 & 9 \\
\hline 17 & hyper & 70 & 30 \\
\hline 20 & hyper & 100 & 1 \\
\hline
\end{tabular}

${ }^{a}$ Peritoneal lavage was performed immediately after death in the two animals that died normoglycaemic of a technical complication (b). In the other eight recipients it was performed approximately 1 or 2 weeks after reoccurrence of hyperglycaemia.

${ }^{\mathrm{b}}$ Died accidentally of a technical complication 


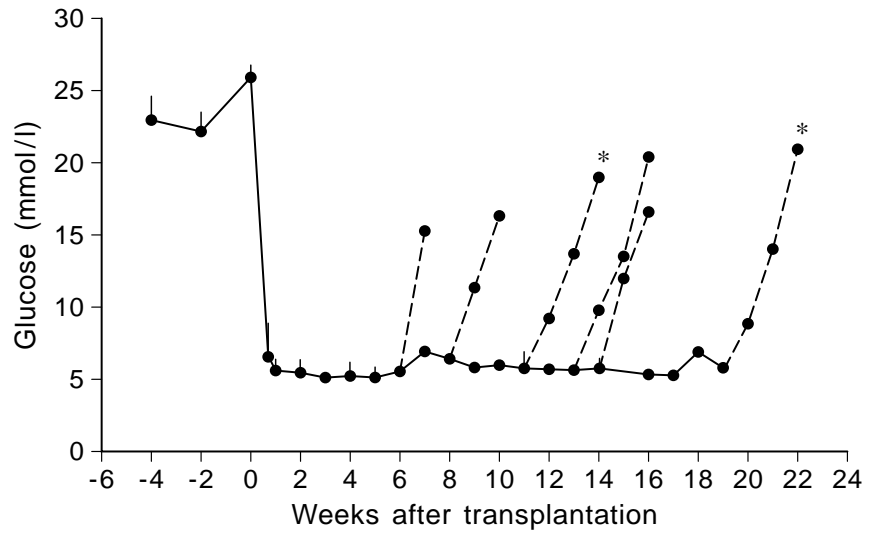

Fig. 7. Non-fasting blood glucose concentrations in streptozotocin diabetic AO-rats $(n=6)$ after implantation of microencapsulated islet isograft. (---) animals returning to hyperglycaemia, * animals with $5-10 \%$ vital beta cells in their native pancreas. Values represent mean $\pm \mathrm{SEM}$

Occasionally, we found some viable islets with large necrotic zones (Fig. 6.B).

Islet isografts encapsulated with purified alginate. In order to exclude that an immune response against the allogeneic islets in the capsules was the cause of the limited graft function we transplanted encapsulated islets isogenically in diabetic AO-rats. Six of eight recipients became normoglycaemic. The recipients of successful isografts showed individual graft survival times quite similar to recipients of allografts (Fig. 7). As shown in Table 2, not only the mean graft survival was similar with encapsulated iso- and allografts, but also the percentage of capsules retrievable by peritoneal lavage and the portion of overgrown capsules. After graft failure the islets in the non-overgrown capsules had a necrotic appearance similar to that observed in allografted encapsulated islets.

\section{Discussion}

This study evaluates the effect of a multi-step alginate purification procedure principally composed of filtration, precipitation, and subsequent protein extraction. This evaluation was not performed by testing the purity of alginate with physical or chemical methods, since it is not known which contaminants are responsible for the reaction against capsules prepared of crude alginate, nor which grade of purity is required to obtain biocompatible capsules. Therefore, we have rather evaluated the effect of purification by assessing the responses to empty and islet containing capsules implanted in the peritoneal cavity of rats.

This is the first study to demonstrate that purification of alginate improves the biocompatibility of alginate-PLL capsules. Nevertheless, even with purified alginate, a small portion of the capsules was always found to be overgrown by fibrous tissue. This indicates that bioincompatibility is influenced not only by the chemical composition but also by individual imperfections of capsules. This is corroborated by our observation that some capsules contain localized defects [30,31].

Islet allografts encapsulated in capsules prepared from purified alginate could successfully be transplanted into streptozotocin diabetic AO-rats. However, the graft function was of limited duration. This limited graft function was not caused by insufficient immunoprotection of the allogenic tissue since alloand isografts had similar graft survival rates. Neither can it be explained by fibrosis of the capsules since the percentage of capsules overgrown was $30 \%$ at maximum and usually below $10 \%$, both in functioning and non-functioning grafts.

One may hypothesize that cytokines released during the cascade of the fibrotic reaction against a small percentage of the capsules can be held responsible for damaging not only the islets in these capsules but also those contained by the other, intact, capsules, thereby inducing graft failure. This, however, is unlikely since we found high numbers of well granulated and intact islets without signs of cytotoxic damage in still normoglycaemic animals 1 month after implantation. At this time, the capsular reaction was completed since an increase was not observed during the following months (Fig.1). Also, we would have expected a more severe fibrotic reaction to be associated with a shorter duration of graft function, but such an association was not observed (Table 1 ).

The phenomenon of graft failure in the absence of overgrowth of the capsules has been reported previously $[1,32,33]$ and is explained by exhaustion of the graft as a consequence of an insufficient number of transplanted encapsulated islets in relation to the glycaemic load. This too, however, appears unlikely to explain the limited graft survival in our study since we transplanted a sufficient islet volume of $10 \mu \mathrm{l}$,

Table 2. Weeks of graft survival, implanted islet volume, recovery rates and percentage of capsules with overgrowth after implantation of microencapsulated rat islet iso- and allografts

\begin{tabular}{lcllll}
\hline $\begin{array}{l}\text { Encapsulated } \\
\text { transplant }\end{array}$ & $\mathrm{n}$ & $\begin{array}{l}\text { Graft survival } \\
(\text { weeks) }\end{array}$ & $\begin{array}{l}\text { Implanted islet volume } \\
(\mu \mathrm{l})\end{array}$ & $\begin{array}{l}\text { Percentage } \\
\text { of recovery }\end{array}$ & $\begin{array}{l}\text { Percentage } \\
\text { of overgrowth }\end{array}$ \\
\hline $\begin{array}{l}\text { Isograft } \\
\text { Allograft }\end{array}$ & 6 & $11.7 \pm 1.8$ & $11.8 \pm 0.8$ & $82.0 \pm 8.1$ & $10.7 \pm 1.9$ \\
\hline
\end{tabular}

a Two recipients which died of technical complications were excluded 
which is equivalent to the volume of islets present in the normal pancreas. Another discrepancy which does not corroborate the explanation of exhaustion is that glucose levels in the peritoneal fluid are approximately $80 \%$ of the ambient intravenous levels [34]. Since the mean of maximum blood glucose concentrations in response to a physiological challenge like a meal is $8.8 \pm 0.4 \mathrm{mmol} / \mathrm{l}$, encapsulated islets in the peritoneal cavity are exposed to a maximum glucose level of approximately $7.0 \mathrm{mmol} / \mathrm{l}$, which cannot induce exhaustion.

The main factor responsible for the limited duration of encapsulated graft function appears to be that the lifespan of encapsulated islets is limited by the presence of the capsule as such, in combination with the intraperitoneal transplantation site. This is quite plausible since a successful transplant of $5 \mu \mathrm{l}$ non-encapsulated islets under the kidney capsule induces permanent normoglycaemia [13], while successful encapsulated islet grafts do not.

If successful, non-encapsulated islets will become completely vascularized after implantation, but microencapsulated islets stay unattached and freely floating in the peritoneal cavity without any vascular connection. This implies that the supply of nutrients, growth factors and substances essential for the homeostasis of islet cells depends upon diffusion from blood to the peritoneal cavity [35] while it may also be delayed by the capsule membrane. Some substances like transferrin may even be retained as the consequence of its incompatible conformation or its large molecular weight. Furthermore, the low oxygen tension [36-38] and low concentrations of essential minerals [36-39] in the peritoneal fluid may also contribute to a gradual decrease of the size of the islet graft and, therefore, limit the lifespan of the graft. The significance of the lack of vascular access for the viability of the encapsulated islets and for the occurrence of graft failure is illustrated by our observations that the diameters of encapsulated islets become smaller with time and that islets gradually develop necrotic zones.

Not only lifespan limitations of encapsulated islet grafts but also their functional limitation may to a large extent be explained by the presence of the capsules as such in combination with the intraperitoneal transplantation site. When diabetic AO-recipients of $10 \mu \mathrm{l}$ of intraperitoneal but non-encapsulated islet isografts were tested at 4 to 6 weeks after transplantation that is after revascularization of the graft, almost normal glucose tolerance and clearcut insulin responses were found [40]. But with successful encapsulated islet grafts such revascularization is lacking and, consequently, the insulin response depends on the transfer of glucose and insulin between the peritoneal fluid and blood. As we previously showed, this transfer is a rather slow process [35] which, together with the barrier effect of the microcapsule, predictably results in strongly delayed responses of plasma insulin and glucose levels when compared to responses by vascularized islets.

Two recipients showed higher peak levels and a longer duration of hyperglycaemia than the other five recipients. This cannot be explained by diminished insulin secretion by the encapsulated islet graft as, remarkably, insulin levels in these two recipients were similar to insulin levels in the other five recipients (Fig.3). Our findings rather suggest insulin insensitivity to have developed in these two animals.

Although purification of alginate improves the biocompatibility of alginate-PLL capsules and thereby deletes one of the major obstacles in the clinical applicability of microencapsulated islets, the problem of limited graft survival remains to be solved. Possible approaches in order to prolong the function of the graft may be transplantation of larger quantities of islets [6], co-encapsulation of cells producing growth factors [41], or transplantation of islets into sites better vascularized than the unmodified peritoneal cavity warranting adequate supply of nutrients and growth factors.

A cknowledgements. This work was supported by a grant from the Diabetes Research Fund of The Netherlands. The author thanks Dr. B.J Thu and Professor Dr. G. Skjåk-Bræk (Nobipol, Department of Biotechnology, University of Trondheim, Norway) for the nuclear magnetic resonance-analysis of the alginate.

\section{References}

1. O'Shea GM, Sun AM (1986) Encapsulation of rat islets of Langerhans prolongs xenograft survival in diabetic mice. Diabetes 35: 943-946

2. Fan MY, Lum ZP, Fu XW, Levesque L, Tai IT, Sun AM (1990) Reversal of diabetes in BB rats by transplantation of encapsulated pancreatic islets. Diabetes 39: 519-522

3. Soon-Shiong P, Feldman E, Nelson R et al. (1992) Successful reversal of spontaneous diabetes in dogs by intraperitoneal microencapsulated islets. Transplantation 5: 769774

4. Cole DR, Waterfall M, McIntyre M, Baird JD (1992) Microencapsulated islet grafts in the $\mathrm{BB} / \mathrm{E}$ rat: a possible role for cytokines in graft failure. Diabetologia 35: 231-237

5. Fritschy WM, De Vos P, Groen H et al. (1994) The capsular overgrowth on microencapsulated pancreatic islet grafts in streptozotocin and autoimmune diabetic rats. Transplant Int 7: 264-271

6. Fritschy WM, Strubbe JH, Wolters GHJ, Van Schilfgaarde R (1991) Glucose tolerance and plasma insulin response to intravenous glucose infusion and test meal in rats with microencapsulated islet allografts. Diabetologia 34: 542547

7. Soon-Shiong P, Otterlie M, Skjåk-Bræk G et al. (1991) An immunological basis for the fibrotic reaction to implanted microcapsules. Transplant Proc 23: 758-759

8. Clayton HA, London NJM, Colloby PS, Bell PRF, James RFL (1991) The effect of capsule composition on the biocompatibility of alginate-poly-l-lysine capsules. J Microencapsulation 8: 221-233 
9. Zimmermann U, Klöck G, Federlin K et al. (1992) Production of mitogen-contamination free alginates with variable ratios of mannuronic acid to guluronic acid by free flow electrophoresis. Electrophoresis 13: 269-274

10. Gotfredsen CF, Stewart MG, O'Shea GM, Vose JR, Horn T, Moody AJ (1990) The fate of transplanted encapsulated islets in spontaneously diabetic BB/WOR rats. Diabetes Research 15: 157-163

11. Vandenbossche GM, Bracke ME, Cuvelier CA, Bortier HE, Mareel MM, Remon JP (1993) Host reaction against empty alginate-polylysine microcapsules. Influence of preparation procedure. J Pharm Pharmacol 45: 115-120

12. Skjåk-Bræk G, Martinsen A (1991) Applications of some algal polysaccharides in biotechnology. In: MD Guiry, G Blunden (eds) vs eaweed resources in Europe: uses and potentials. Wiley \& Sons, Chichester, pp 219-230

13. Suylichem van PTR, Strubbe JH, Houwing H, Wolters GHJ, Van Schilfgaarde R (1994) Rat islet isograft function: effect of graft volume and transplantation site. Transplantation 57: 1010-1017

14. Strubbe JH, Bouman PR (1978) Plasma insulin patterns in the unanesthetized rat during intracardiac infusion and spontaneous ingestion of graded loads of glucose. Metabolism 27: 341-351

15. Steffens AB (1969) A method for frequent sampling of blood and continuous infusion of fluids in the rat without disturbing the animal. Physiol Behav 4: 833-836

16. Houwing H, Benthem L, Van Suylichem PTR, Van der Leest J, Strubbe JH, Steffens AB (1995) Islet transplantation in diabetic rats normalizes basal and exercise-induced energy metabolism. Diabetologia 38: 919-926

17. Wolters GHJ, Van Suylichem PTR, Van Deynen JHM, van Schilfgaarde R (1990) Factors influencing the isolation process of islets of Langerhans. Horm Metab Res 25: 20-26

18. Fritschy WM, Van Suylichem PTR, Wolters GHJ, Van Schilfgaarde R (1992) Comparison of top and bottom loading of a dextran gradient for rat pancreatic islet purification. Diabetes Research 19: 91-95

19. Haug A, Larsen B (1963) The solubility of alginate at low pH. Acta Chem Scand 17: 1653-1662

20. Staub AM (1965) Removal of proteins. In: RL Whistler, JN BeMiller, ML Wolfrom (eds) Methods in carbohydrate chemistry, Vol. 5, New York, London, Academic Press, pp 5

21. Haug A (1965) Alginic acid. Isolation and fractionation with potassium chloride and manganous ions. In: RL Whistler, JN BeMiller, ML Wolfrom (eds) Methods in carbohydrate chemistry, Vol. 5, New York, London, Academic Press, pp 69-72

22. Haug A, Myklestad S, Larsen B, Smidsrød O (1967) Correlation between chemical structure and physical properties of alginates. Acta Chem Scand 21: 768-778

23. Thu B, Bruheim P, Espevik T, Smidsrød O, Soon-Shiong P, Skjåk-Braek G (1996) Alginate polycation microcapsules.1. Interaction between alginate and polycation. Biomaterials 17: 1031-1040

24. Thu B, Bruheim P, Espevik T, Smidsrød O, Soon-Shiong P, Skjåk-Braek G (1996) Alginate polycation microcapsules.2. Some functional properties. Biomaterials 17: 10691079

25. Wolters GHJ, Fritschy WM, Gerrits D, Van Schilfgaarde R (1992) A versatile alginate droplet generator applicable for microencapsulation of pancreatic islets. J Applied Biomater 3: 281-286

26. Fritschy WM, Wolters GHJ, Van Schilfgaarde R (1991) Effect of alginate-polylysine-alginate microencapsulation on in vitro insulin release from pancreatic islets. Diabetes 40: 37-43

27. Vos de P, Haan de BJ, Pater J, Van Schilfgaarde R (1996) Association between capsule diameter, adequacy of encapsulation, and survival of microencapsulated rat islet allografts. Transplantation 62: 893-899

28. Bank HL (1988) Rapid assessment of islet viability with acridine orange and propidium iodide. In Vitro Cell Dev Biol 24: 266-273

29. Gerrits PO, Horobin RW, Wright DJ (1990) Staining sections of water miscible resins. 1. Effects of the molecular size of stain, and of resin cross-linking, on the staining of glycol methacrylate embedded tissues. J Microsc 69: 279-290

30. Vos de P, Wolters GHJ, Fritschy WM, Van Schilfgaarde R (1993) Obstacles in the application of microencapsulation in islet transplantation. Int J Art Org 16: 205-212

31. De Vos P, Wolters GHJ, Van Schilfgaarde R (1994) Possible relationship between overgrowth of alginate-polylysine-alginate microencapsulated islets and the microcapsule integrity. Transplant Proc 26: 782-783

32. Lum ZP, Krestow M, Tai IT, Vacek I, Sun AM (1992) Xenografts of rat islets into diabetic mice. Transplantation 53: $1180-1183$

33. Sun YL, Ma X, Zhou D, Vacek I, Sun AM (1993) Porcine pancreatic islets: isolation, microencapsulation, and xenotransplantation. Artif Organs 17: 727-733

34. Velho G, Froguel P, Reach G (1989) Determination of peritoneal glucose kinetics in rats: implications for the peritoneal implantation of closed-loop insulin delivery systems. Diabetologia 32: 331-336

35. De Vos P, Vegter D, De Haan BJ, Strubbe JH, Bruggink JE, Van Schilfgaarde R (1996) Kinetics of intraperitoneal insulin in rats: functional implications for the bioartificial pancreas. Diabetes 45 (8):1102-1107

36. Colton CK, Avgoustiniatos ES (1991) Bioengineering in development of the hybrid artificial pancreas. J Biomech Eng 113: 152-168

37. Schrezenmeier J, Kirchgessner J, Gerö L, Kunz LA, Beyer J, Mueller-Klieser W (1994) Effect of microencapsulation on oxygen distribution in islet organs. Transplantation 57: 1308-1314

38. Renvall S, Niinikoski J (1975) Intraperitoneal oxygen and carbon dioxide tensions in experimental adhesion disease and peritonitis. Am J Surg 130: 286-292

39. Hsu BRS, Fu SH, Huang YY, Chen HC, Huang HS (1994) Prolonged hyperglycemia in streptozotocin-induced diabetic mice after intraperitoneal treatment with microencapsulated islets. Transplant Proc 26: 3706-3708

40. Fritschy WM, van Straaten JF, De Vos P, Strubbe JH, Wolters GH, Van Schilfgaarde R (1991) The efficacy of intraperitoneal pancreatic islet isografts in the reversal of diabetes in rats. Transplantation 52: 777-783

41. Metrakos P, Yuan S, Agapitos D, Rosenberg L (1993) Intercellular communication and maintenance of islet cell mass - implications for islet transplantation. Surgery 114: 423428 